\title{
ANEMIA EM CRIANCCAS DE RIO BRANCO, AC: PREVALÊNCIA E FATORES ASSOCIADOS
}

\author{
PREVALENCE AND ASSOCIATED FACTORS \\ TO ANAEMIA IN CHILDREN
}

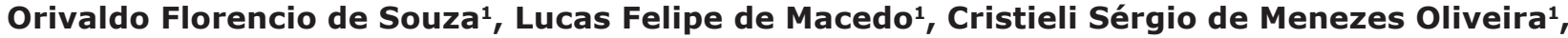 \\ Thiago Santos de Araújo ${ }^{1}$, Pascoal Torres Muniz ${ }^{1}$
}

\begin{abstract}
RESUMO:
Objetivos: Analisar a prevalência e os fatores associados à anemia em crianças de Rio Branco, Acre. Métodos: Participaram do estudo 610 crianças de 6 a 59 meses. Foi aplicado um questionário com questões sobre as características da mãe, práticas alimentares e morbidades pregressas. 0 diagnóstico de anemia baseou-se no valor de hemoglobina de $110 \mathrm{~g} / \mathrm{l}$. Na análise dos fatores associados foi utilizada a regressão de Poisson múltipla, com entrada das variáveis em blocos hierarquizados. Resultados: A prevalência de anemia foi de $51,8 \%$. Os fatores associados à anemia foram: idade abaixo de 24 meses: (razão de prevalência [RP]: 1,51; intervalo de confiança em 95\% [IC95\%]: 1,17-1,95); anos de estudo da mãe entre 5-8 anos (RP: 1,$34 ;$ IC95\%: 1,13-1,58) e igual ou inferior a 4 anos (RP: 1,32; IC95\%: 1,05-1,65); situação de anemia da mãe (RP: 1,28; IC95\%: 1,08-1,51); freqüência de consumo em menos de uma vez por semana de carnes (RP: 1,35 ; IC95\%: 1,03-1,77) e frutas (RP: 1,28; IC95\%: 1,09-1,52). Conclusões: A prevalência de anemia em crianças de Rio Branco é um problema grave em saúde pública. Destaca-se a necessidade do fortalecimento de políticas em atenção à saúde da família e ao aconselhamento sobre as práticas alimentares voltadas a mãe.
\end{abstract}

Palavras-chave: anemia; saúde da criança; estado nutricional; estudos transversais; fatores epidemiológicos; prevalência.

\begin{abstract}
:
Objectives: To analyse the prevalence and factors associated with anaemia in children from Rio Branco, Acre. Methods: The study included 610 children between 6 and 59 months old. A questionnaire was issued with questions on characteristics of the mother, feeding practices and antecedent morbidity. The anaemia diagnosis was based on haemoglobin levels at $110 \mathrm{~g} / \mathrm{l}$. Multiple Poisson regression was used to analyse the associated factors with variables entered into hierarchical blocks. Results: The anaemia prevalence was $51.8 \%$. The factors associated with anaemia were the following: age below 24 months (prevalence ratio [PR]: 1.51 and 95\% confidence interval [95\% CI]: 1.17 to 1.95 ); years of formal education for the mother between $5-8$ years (PR: 1.34 and $95 \%$ CI: 1.13 to 1.58 ) and less than or equal to 4 years (PR: 1.32 and $95 \% \mathrm{CI}: 1.05$ to 1.65 ); anaemia in the mother (PR: 1.28 and $95 \% \mathrm{CI}: 1.08$ to 1.51 ); and consumption frequency less than once a week for meats (PR: 1.35 and 95\% CI: 1.03 to 1.77 ) and fruits (PR: 1.28 and $95 \%$ CI: 1.09 to 1.52 ). Conclusions: The prevalence of anaemia among children in Rio Branco is a serious public health problem. The results highlight the need to strengthen policies on family health care and provide mothers with counselling on feeding practices.
\end{abstract}

Key words: anemia; child health; nutritional status; cross-sectional studies; epidemiologic factors; prevalence.

1 Centro de Ciências da Saúde e do Desporto da Universidade Federal do Acre Corresponding author: orivaldofs.ufac@gmail.com

Suggested citation: de Souza OF, et al. Prevalence and associated factors to anaemia in children . J. Hum. Growth Dev. 2012; 22(3): 307-313

Manuscript submitted Dec 08 2011, accepted for publication Aug 22012. 


\section{INTRODUÇÃO}

A anemia é problema mundial em saúde pública, coexistindo com outras patologias, tais como as carências de micronutrientes e malária ${ }^{1}$. As crianças menores de 5 anos e as mulheres em idade reprodutiva representam os grupos mais vulneráveis à ocorrência da anemia ${ }^{1,2}$. Segundo relatório da Organização Mundial da Saúde ${ }^{2}(\mathrm{OMS})$, existe cerca de 293,1 milhões de crianças menores de 5 anos anêmicas em todo o mundo, com prevalência mundial estimada em 47,4\%.

Apesar de evidências atuais demonstrarem declínio da desnutrição em crianças brasileiras ${ }^{3}$, contrariamente, a anemia apresenta aumento de prevalência em várias regiões brasileiras ${ }^{4,5}$. A Pesquisa Nacional de Demografia e Saúde da Criança e da Mulher ${ }^{6}$ de 2006 revelou prevalência de anemia de $20,9 \%$ para crianças brasileiras. Especificamente na Região Norte havia $10,4 \%$ de crianças anêmicas. Contudo, investigações realizadas em vários estados brasileiros revelaram prevalências maiores de anemia5,7. Em cidades no estado do Acre, duas investigações de base populacional em crianças menores de 5 anos mostraram prevalência de anemia acima de 20,0\%

Conforme apontam investigações nacionais e internacionais, a anemia em crianças menores de 5 anos está associada a vários fatores socioeconômicos, características maternas, consumo alimentar e morbidades pregressas ${ }^{10}$. No entanto, esses fatores apresentam-se diferenciados quanto ao contexto geográfico. Logo, é necessária a identificação dos fatores associados à anemia de crianças de modo regionalizado para programar ações de prevenção e controle da anemia com efetividade.

Devido a importância da anemia em contexto mundial e nacional e a carência de estudos relacionados ao problema na capital acreana, o objetivo dessa investigação foi analisar a prevalência e os fatores associados à anemia em crianças de 6 até 59 meses de Rio Branco, Acre.

\section{MÉTODO}

Trata-se de um estudo transversal, com amostragem probabilística, realizado no primeiro semestre de 2008. Participaram do estudo crianças de 6 a 59 meses residentes no município de Rio Branco (latitude: 9 58'29"; longitude: 69 48' 36"; altitude: 153 metros), estado do Acre, Brasil. Esse município tem Índice de Desenvolvimento Infantil de 0,639, estando este valor próximo da média nacional de $0,667^{11}$.

A amostragem por conglomerados foi realizada em duas fases sendo a primeira constituída pelo sorteio de 34 setores dentre os 250 existentes no município e a segunda do sorteio de 75 domicílios em cada setor selecionado. Dentre os 2550 domicílios sorteados, 622 domicílios compuseram a amostra por terem entre seus residentes crianças com idade entre 6 e 59 meses.
O critério de exclusão foi apresentar manifestações clínicas que impossibilitasse a realização da coleta de sangue. Todas as crianças diagnosticadas com anemia foram enviadas para os serviços de assistência à saúde do município de Rio Branco. O estudo foi aprovado pelo Comitê de Ética em Pesquisa da Universidade Federal do Acre (Processo $n^{\circ}$ 23107.001150/2007-22).

A coleta de dados foi realizada por profissionais e acadêmicos da área da saúde que participaram de sessões treinamento. Questionário estruturado foi aplicado à mãe biológica da criança no interior dos domicílios sorteados. No caso da mãe biológica não habitar no domicílio com a criança, as informações foram coletadas do cuidador do sexo feminino responsável pela criança.

As informações coletadas sobre as crianças foram: características demográficas, aleitamento materno exclusivo, práticas alimentares, peso ao nascer e morbidades nos últimos 15 dias anteriores à entrevista referidas pela mãe. As faixas etárias foram categorizadas em: 6 e 23 meses, 24 e 47 meses e 48 e 59 meses. O aleitamento materno exclusivo foi definido em menor ou igual a 180 dias e acima de 180 dias. Enquanto que a variável peso ao nascer foi categorizada em baixo peso ao nascer (menor ou igual a $2.500 \mathrm{~g}$ ) e peso adequado ao nascer (acima de $2.500 \mathrm{~g}$ ).

As práticas alimentares foram identificadas por questões sobre a frequência semanal de ingestão de carnes, feijão, hortaliças e frutas. Para análise dos dados, todas essas variáveis foram categorizadas em: consumo inferior a 1 vez por semana e igual ou mais a 1 vez na semana.

As informações maternas investigadas foram: idade, anos de estudo, situação ocupacional e convivência com companheiro. A idade da mãe foi categorizada em menor ou igual a 20 anos e maior de 20 anos. Os anos de estudo foram categorizados em três categorias (0-4 anos, 5-8 anos e 9 ou mais anos). A variável situação ocupacional mãe foi constituída pelas categorias: trabalha (atividades remuneradas) e não trabalha. Enquanto que o estado marital da mãe foi categorizado em: sem companheiro (solteira, separada ou viúva) e com companheiro (casada ou em união consensual).

A estatura e o comprimento corporal das crianças foram medidos por estadiômetro e antropômetro infantil, respectivamente, ambos com precisão de $0,1 \mathrm{~cm}$ e utilizando os procedimentos preconizados pela OMS ${ }^{12}$. Com auxílio do programa WHO Anthro 2005, o escore Z do índice estatura para idade foi obtido a partir do padrão de crescimento da criança da OMS de $2006^{13}$. Utilizou-se o ponto de corte igual ou menor a -2 escore $Z$ para a classificação de déficit estatural ${ }^{12}$. As morbidades pregressas investigadas foram referentes ao acometimento de diarréia e a ocorrência de sangue nas fezes nos últimos 15 dias antes da entrevista. As categorias para ambas variáveis foram ausência e presença. 
Uma amostra de gota de sangue obtida após puncionar um dedo da mão foi utilizada para a quantificação da concentração de hemoglobina mediante um hemoglobinômetro portátil (Hemocue ${ }^{\circledR}$, Ängelhom, Suécia). Conforme estabelecido pela $\mathrm{OMS}^{1}$, o diagnóstico de anemia foi definido pelos pontos de corte inferior a $110 \mathrm{~g} / \mathrm{l}$ de hemoglobina para as crianças e inferior a $120 \mathrm{~g} / \mathrm{l}$ de hemoglobina para as mães.

Seguindo o procedimento de dupla entrada, os dados foram digitados no programa Epi-Info 6.01. Por conseguinte, os dados foram transferidos para o programa estatístico Stata ${ }^{T M}$ 9.2. Os fatores associados à anemia foram analisados em duas etapas. Inicialmente, as variáveis independentes que apresentaram associações com a variável anemia valor de $p<0,20$ (teste de qui-quadrado para heterogeneidade) foram selecionadas para compor os modelos múltiplos. Posteriormente, foram identificados os fatores associados à anemia mediante análise múltipla e hierarquizada de regressão de Poisson ${ }^{14,15}$. Adotou-se o seguinte modelo conceitual adaptado de Osório ${ }^{16}$ para a entrada das variáveis na análise dos dados: $1^{\circ}$ bloco (características da mãe); $2^{\circ}$ bloco (práticas alimentares); $3^{\circ}$ bloco (peso ao nascer); e, $4^{\circ}$ bloco (estatura para idade e morbidades pregressas). As variáveis sexo e idade das crianças foram in- troduzidas no primeiro bloco e permaneceram nas etapas subseqüentes.

\section{RESULTADOS}

Do total das 622 crianças encontradas nos domicílios dos setores sorteados, foi realizada a quantificação da concentração de hemoglobina em 610 crianças ( $98 \%$ dos elegíveis). As perdas ocorreram por recusa dos responsáveis na participação das crianças na pesquisa. Das crianças elegíveis, $50,3 \%$ e $49,7 \%$ eram do sexo masculino e feminino, respectivamente.

A prevalência geral de anemia foi de 51,8\% para as crianças de 6 até 59 meses de Rio Branco, Acre. Prevalências altas de anemia foram identificadas nas faixas etárias menores em ambos os sexos (Figura 1). Na faixa etária dos 6 aos 11 meses, identificou-se as prevalências de anemia mais elevadas, sendo de $65,7 \%$ e $87,8 \%$ para os sexos masculino e feminino, respectivamente.

A prevalência de anemia por características da mãe, aleitamento materno exclusivo, peso ao nascer, estado nutricional e morbidades pregressas estão apresentadas nas Tabelas 1 e 2.

$\mathrm{Na}$ análise dos fatores associados, foi verificado que as crianças com idade abaixo de 24 meses tinham

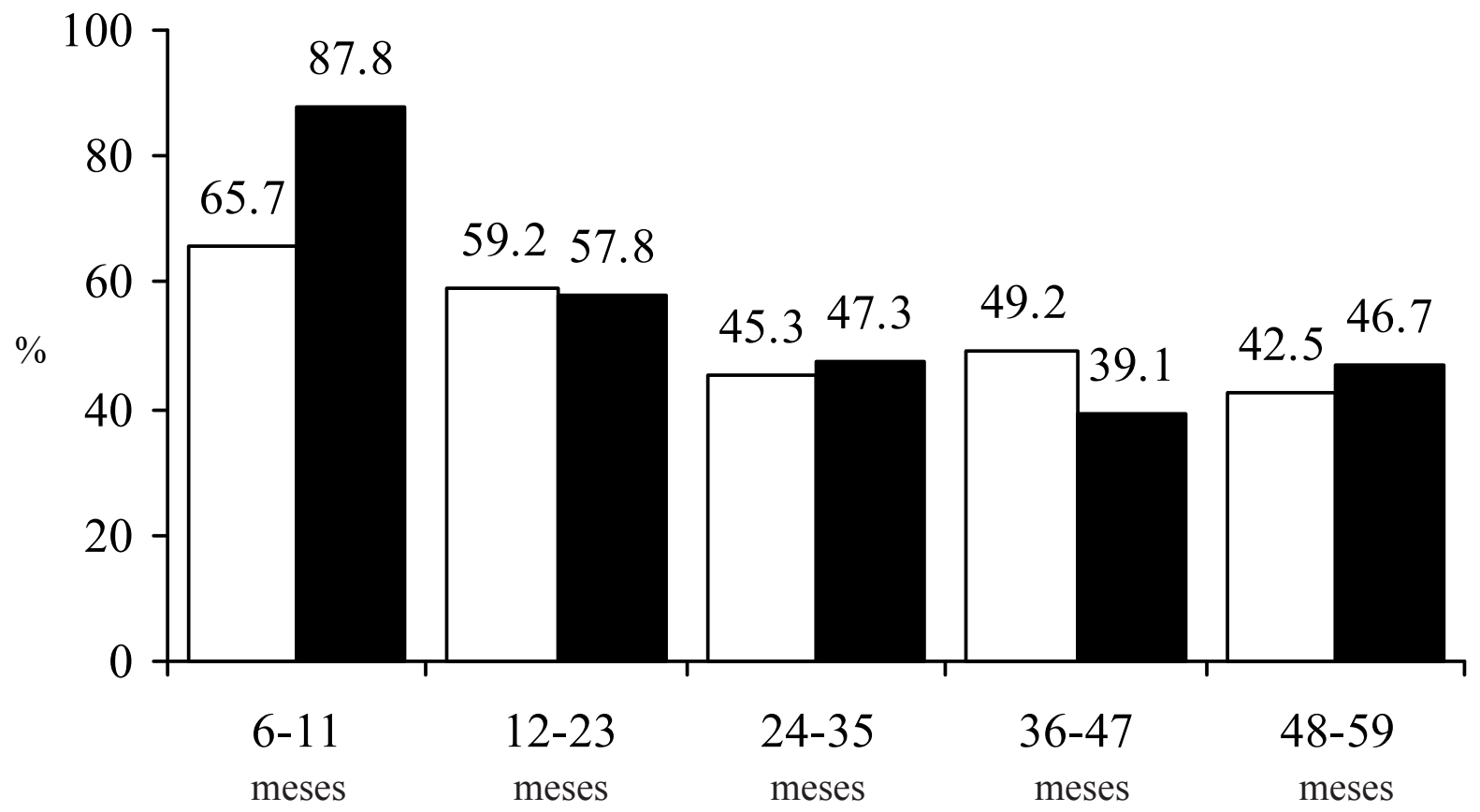

masc. Feminino

Gráfico 1: Prevalência de anemia por sexo e faixa etária de crianças de Rio Branco, AC, 2008 
1,51 vezes mais anemia em relação às crianças maiores. As crianças cujas mães tinham 8 anos ou menos de estudo apresentavam maior suscetibilidade ao aco- metimento de anemia. Também a situação da mãe estar com anemia aumentava em 1,28 vezes a prevalência de anemia da criança (Tabela 3).

Tabela 1: Prevalência e razão de prevalência da anemia segundo características da mãe das crianças de Rio Branco/AC, 2008

\begin{tabular}{|c|c|c|c|c|c|}
\hline & $\mathbf{n}$ & $\%$ & $\begin{array}{l}\text { Razão de } \\
\text { Prevalência }\end{array}$ & IC $95 \%$ & $\mathbf{p}$ \\
\hline \multicolumn{6}{|l|}{ Sexo } \\
\hline Masculino & 308 & 51,6 & 1 & & \\
\hline Feminino & 302 & 51,9 & 1,00 & $(0,87 ; 1,16)$ & 0,922 \\
\hline \multicolumn{6}{|l|}{ Idade } \\
\hline $48-59$ meses & 124 & 42,7 & 1 & & \\
\hline $24-47$ meses & 287 & 46,6 & 1,09 & $(0,83 ; 1,42)$ & 0,500 \\
\hline $6-23$ meses & 199 & 64,8 & 1,51 & $(1,17 ; 1,95)$ & 0,002 \\
\hline \multicolumn{6}{|l|}{ Idade da mãe } \\
\hline Maior de 20 anos & 505 & 50,1 & 1 & & \\
\hline Igual ou menor de 20 anos & 101 & 59,4 & 1,18 & $(0,97 ; 1,44)$ & 0,089 \\
\hline \multicolumn{6}{|l|}{ Anos de estudos da mãe } \\
\hline 9 anos ou mais & 302 & 44,0 & 1 & & \\
\hline $5-8$ anos & 192 & 59,9 & 1,36 & $(1,15 ; 1,60)$ & 0,001 \\
\hline $0-4$ anos & 109 & 57,8 & 1,31 & $(1,03 ; 1,66)$ & 0,027 \\
\hline \multicolumn{6}{|l|}{ Situação ocupacional da mãe } \\
\hline Trabalha & 418 & 51,9 & 1 & & \\
\hline Não trabalha & 190 & 51,5 & 0,99 & $(0,86 ; 1,14)$ & 0,927 \\
\hline \multicolumn{6}{|l|}{ Estado marital da mãe } \\
\hline Com companheiro & 438 & 50,2 & 1 & & \\
\hline Sem companheiro & 169 & 56,2 & 1,11 & $(0,95 ; 1,31)$ & 0,165 \\
\hline \multicolumn{6}{|l|}{ Situação de anemia da mãe } \\
\hline Não anêmica & 378 & 46,5 & 1 & & \\
\hline Anêmica & 215 & 60,0 & 1,28 & $(1,07 ; 1,53)$ & 0,007 \\
\hline
\end{tabular}

Tabela 2: Prevalência e razão de prevalência da anemia segundo aleitamento materno, frequência de consumo de alimentos, peso ao nascer, morbidades pregressas e déficit estatural de crianças de Rio Branco/AC, 2008

\begin{tabular}{|c|c|c|c|c|c|}
\hline & $\mathrm{n}$ & $\%$ & Razão de Prevalência & IC95\% & $\mathrm{p}$ \\
\hline \multicolumn{6}{|l|}{ Aleitamento exclusivo } \\
\hline 180 dias ou mais & 235 & 51,0 & 1 & & \\
\hline Menor de 180 dias & 358 & 51,9 & 1,01 & $(0,87 ; 1,17)$ & 0,813 \\
\hline \multicolumn{6}{|l|}{ Frequência de consumo de carnes } \\
\hline 1 vez ou mais na semana & 93 & 34,4 & 1 & & \\
\hline Inferior a $1 \mathrm{vez}$ por semana & 444 & 52,2 & 1,51 & $(1,13 ; 2,02)$ & 0,006 \\
\hline \multicolumn{6}{|l|}{ Freqüência de feijão } \\
\hline 1 vez ou mais na semana & 448 & 46,6 & 1 & & \\
\hline Inferior a 1 vez por semana & 90 & 62,2 & 1,33 & $(1,09 ; 1,62)$ & 0,006 \\
\hline \multicolumn{6}{|c|}{ Frequência de consumo de hortaliças } \\
\hline 1 vez ou mais na semana & 172 & 45,3 & 1 & & \\
\hline Inferior a 1 vez por semana & 365 & 50,9 & 1,12 & $(0,91 ; 1,38)$ & 0,261 \\
\hline \multicolumn{6}{|l|}{ Frequência de consumo de frutas } \\
\hline 1 vez ou mais na semana & 419 & 44,8 & 1 & & \\
\hline Inferior a 1 vez por semana & 118 & 64,4 & 1,43 & $(1,23 ; 1,67)$ & 0,000 \\
\hline \multicolumn{6}{|l|}{ Peso ao nascer } \\
\hline Acima de $2.500 \mathrm{~g}$ & 543 & 51,7 & 1 & & \\
\hline Igual ou menor de $2.500 \mathrm{~g}$ & 48 & 54,1 & 1,04 & $(0,75 ; 1,45)$ & 0,781 \\
\hline \multicolumn{6}{|l|}{ Diarréia nos últimos 15 dias } \\
\hline Ausência & 455 & 49,2 & 1 & & \\
\hline Presença & 154 & 59,0 & 1,20 & $(0,99 ; 1,44)$ & 0,054 \\
\hline \multicolumn{6}{|c|}{ Sangue nas fezes nos últimos 15 dias } \\
\hline Ausência & 581 & 51,1 & 1 & & \\
\hline Presença & 29 & 65,5 & 1,28 & $(0,95 ; 1,72)$ & 0,098 \\
\hline \multicolumn{6}{|l|}{ Estatura para idade } \\
\hline Adequado & 547 & 50,8 & 1 & & \\
\hline Déficit estatural & 41 & 68,2 & 1,34 & $(1,07 ; 1,67)$ & 0,010 \\
\hline
\end{tabular}


Tabela 3: Fatores associados à anemia em crianças de Rio Branco/AC, 2008

\begin{tabular}{|c|c|c|c|}
\hline & Razão de Prevalência Ajustada & IC95\% & $p$ \\
\hline \multicolumn{4}{|c|}{ ( } \\
\hline Masculino & 1 & & \\
\hline Feminino & 1,02 & $(0,88 ; 1,18)$ & 0,758 \\
\hline \multicolumn{4}{|l|}{ Idade } \\
\hline $48-59$ meses & 1 & & \\
\hline $24-47$ meses & 1,09 & $(0,84 ; 1,42)$ & 0,494 \\
\hline $6-23$ meses & 1,51 & $(1,17 ; 1,95)$ & 0,002 \\
\hline \multicolumn{4}{|l|}{ Anos de estudo da mãe * } \\
\hline 9 anos ou mais & 1 & & \\
\hline $5-8$ anos & 1,34 & $(1,13 ; 1,58)$ & 0,001 \\
\hline $0-4$ anos & 1,32 & $(1,05 ; 1,65)$ & 0,017 \\
\hline \multicolumn{4}{|l|}{ Situação de anemia da mãe * } \\
\hline Não anêmica & 1 & & \\
\hline Anêmica & 1,28 & $(1,08 ; 1,51)$ & 0,005 \\
\hline \multicolumn{4}{|c|}{ Freqüência de consumo de carnes $†$} \\
\hline 1 vez ou mais na semana & 1 & & \\
\hline Inferior a 1 vez por semana & 1,35 & $(1,03 ; 1,77)$ & 0,027 \\
\hline \multicolumn{4}{|c|}{ Freqüência de consumo de frutas $†$} \\
\hline 1 vez ou mais na semana & 1 & & \\
\hline Inferior a $1 \mathrm{vez}$ por semana & 1,28 & $(1,09 ; 1,52)$ & 0,004 \\
\hline
\end{tabular}

* Ajustado: idade e sexo;

† Ajustado: idade, sexo, anos de estudo da mãe e situação de anemia da mãe.

\section{DISCUSSÃo}

No município de Rio Branco foi identificada alta prevalência geral de anemia de 51,8\% em crianças de 6 a 59 meses, sendo considerado um problema grave em saúde pública. A ocorrência da anemia em crianças de Rio Branco está associada às variáveis do contexto sócio-familiar e a freqüência de consumo de alimentos ricos em ferro e estimuladores da absorção do ferro inferior a uma vez por semana.

Essa alarmante magnitude de crianças de Rio Branco acometidas pela anemia concorda com levantamentos utilizando delineamentos transversais em diversas regiões brasileiras ${ }^{5,7}$. Investigações sobre a prevalência de anemia em crianças de cidades do interior do estado do Acre que possuem acesso por estrada pavimentada diferem dos valores encontrados em crianças de Rio Branco, com prevalências entre $24,5 \%$ e $36,3 \% 8,17$. Por outro lado, no município de Jordão, sem acesso por via terrestre, identificaram elevada prevalência de anemia de $57,3 \% \%^{9}$. Apesar de haver diferentes magnitudes na prevalência de anemia em crianças na faixa etária de 6 a 59 meses, a situação de gravidade em saúde pública, definido pela prevalência acima de $40 \%^{1}$, também está presente na maioria dos levantamentos brasileiros.

Várias investigações de base populacional e em creches realizadas no Brasil, inclusive no estado do Acre, identificaram maior prevalência de anemia na faixa etária abaixo de 24 meses em contraste as crianças com idades maiores ${ }^{8,10,18,19}$. O término do período de aleitamento materno exclusivo ocorre em faixa etária com alta necessidade de ferro por kilograma de peso corporal, decorrente da aceleração da velocidade de crescimento ${ }^{20}$. Nessa fase há introdução de dieta com baixa biodisponibilidade em ferro ${ }^{21,22}$. Sugere-se que essas circunstâncias, por consequência, podem ter conduzido a elevada prevalência da anemia na faixa etária de 6 a 11 meses em crianças de Rio Branco.

Coincidente aos resultados evidenciados pelo presente estudo, investigações de base populacional realizadas nos estados do Pernambuco e São Paulo identificaram que o menor número de anos de estudo da mãe estava associado com a anemia do filho 22,23 . Inferindo que a escolaridade proporciona melhor entendimento das práticas alimentares, logo o maior número de anos estudados pela mãe pode conduzir ao cuidado alimentar adequado da criança. Por outro lado, a menor escolaridade sugere atuação em trabalho com baixa rentabilidade, fato que proporciona pior acesso aos alimentos e aos bens e serviços de assistência à saúde para à criança ${ }^{16}$.

No presente estudo o acometimento da anemia pela mãe da criança esteve associada à ocorrência de anemia pela criança. Associação semelhante foi evidenciada em investigação realizada no Nordeste brasileiro ${ }^{24}$ e no estado do Acre $^{9}$. A mãe convivendo no mesmo contexto domiciliar da criança compartilham aspectos semelhantes, tais como a pobreza, a baixa biodisponibilidade de ferro e vitamina B12 nos alimentos ou a suscetibilidade a doenças infecciosas. Logo, o contexto sócio-familiar pode estar influenciando concomitantemente a ocorrência de anemia do filho e da mãe. Além disso, existe a possibilidade de anemias de origens hereditárias estarem influenciando, em parte, essa associação ${ }^{25}$.

As práticas alimentares estão associadas à ocorrência de anemia em crianças $^{21,26}$. De modo 
especial, as frutas contêm minerais e vitaminas com efeito estimulador para a absorção de ferro ${ }^{20}$. Considerando todas as capitais de estados brasileiros, dados da Pesquisa de Orçamentos Familiares ${ }^{27}$ de 2002-03 revelaram que o município de Rio Branco tinha a segunda menor disponibilidade domiciliar de frutas e sucos naturais. Consequentemente, essa situação expõe as crianças de Rio Branco ao acometimento de anemia, principalmente por deficiência de ferro.

As carnes apresentam alta biodisponibilidade de ferro heme e apresentam-se como facilitador para a absorção do ferro não-heme contido em hortaliças ${ }^{28,29}$, logo conferindo um efeito protetor para a anemia. Desse modo, as crianças de Rio Branco expostas ao consumo de carnes inferior a uma vez por semana foram mais suscetíveis a anemia. Informações coincidentes foram evidenciadas em estudo de base populacional realizado no estado do Pernambuco ${ }^{22}$, onde o menor consumo de ferro heme apresentava-se associada à ocorrência de anemia em crianças. Do mesmo modo, em ambulatório de Hospital especializado em pediatria no Rio de Janeiro, foi identificado que crianças na faixa etária de 12 a 18 meses com anemia tinham dieta pobre em carnes em relação as crianças sem anemia ${ }^{26}$.

\section{REFERÊNCIAS}

1. WHO (World Health Organization). Iron Deficiency Anaemia. Assessment, Prevention and Control. Geneva: WHO; 2001.

2. Benoist B, McLean E, Egli I, Cogswell M. Worldwide prevalence of anaemia 1993-2005: WHO global database on anaemia. Geneva: WHO; 2008.

3. Monteiro CA, Benicio MHA, Konno SC, Silva ACF, Lima ALL, Conde WL. Causes for the decline in child under-nutrition in Brazil, 1996-2007. Rev Saude Publica. 2009;43:35-43.

4. Batista Filho M, Assis AMO, Kac G. Transição nutricional: conceitos e características. In: Kac G, Sichieri R, Gigante DP, organizadores. Epidemiologia nutricional. Rio de Janeiro: Editora Fiocruz/São Paulo: Editora Atheneu; 2007. p. 445-60.

5. Lira PIC, Ferreira LOC. Epidemiologia da anemia ferropriva. In: Kac G, Schieri R, Gigante DP, organizadores. Epidemiologia nutricional. Rio de Janeiro: Editora Fiocruz/São Paulo: Editora Atheneu; 2007. p. 337-23.

6. Brasil. Ministério da Saúde. Pesquisa Nacional de Demografia e Saúde da Criança e da Mulher - PNDS 2006: dimensões do processo reprodutivo e da saúde da criança. Brasília: Ministério da Saúde; 2009.

7. Jordão RE, Bernardi JLD, Barros Filho AA. Prevalência de anemia ferropriva no Brasil: uma revisão sistemática. Rev Paul Pediatr. 2009;27:90-8.
Em específico para o município de Rio Branco não foram identificadas publicações referentes a efetividade do Programa Nacional de Suplementação de Ferro (PNSF). Entretanto, investigações realizadas em algumas cidades brasileiras evidenciaram fatores que interferem na efetiva implementação da PNSF, tais como: gestão e organização de recursos humanos e materiais ${ }^{30}$ e a baixa sensibilização das famílias quanto à importância da suplementação com sulfato ferroso ${ }^{31,32}$. Em 2008, no município de Rio Branco havia baixa cobertura $(49,6 \%)$ da Estratégia de Saúde da Família ${ }^{33}$. Por consequência, sugere-se que haja implicação na operacionalização do PNSF no município estudado, pelo fato de limitar a distribuição do sulfato ferroso e o acompanhamento da suplementação.

Conclui-se que a prevalência de anemia em crianças de Rio Branco é um problema grave em saúde pública. As crianças cujas mães tinham baixa escolaridade e estavam acometidas pela anemia mostraram-se mais suscetíveis à anemia. As práticas alimentares inadequadas quanto a freqüência de consumo de carnes e frutas inferior a uma vez na semana apresentaram-se associadas à ocorrência de anemia nas crianças de Rio Branco, estado do Acre.

8. Castro TG, Silva-Nunes $M$, Conde $W L$, Muniz PT, Cardoso MA. Anemia e deficiência de ferro em pré-escolares da Amazônia Ocidental brasileira: prevalência e fatores associados. Cad Saude Publica. 2011;27:131-42.

9. Oliveira CC, Cardoso MA, Araújo TS, Muniz PT. Anemia em crianças de 6 a 59 meses e fatores associados no Município de Jordão, Estado do Acre, Brasil. Cad Saude Publica. 2011;27:100820.

10. Leal LP, Osório MM. Fatores associados à ocorrência de anemia em crianças menores de seis anos: uma revisão sistemática dos estudos populacionais. Rev Bras Saude Matern Infant. 2010;10:417-39.

11. UNICEF (Fundo das Nações Unidas para a Infância). Situação da infância brasileira 2006. Brasília: UNICEF; 2005. [acesso em 11 jun 2011]. Disponível em: http://www.unicef.org/ brazil/pt/Pags_001_007_Abre.pdf

12. WHO (World Health Organization). Physical Status: The Use and Interpretation of Anthropometric Indicators of Nutritional Status. Geneva: WHO; 1995. (Technical Report Series 854).

13. WHO (World Health Organization). WHO Multicentre Growth Reference Study Group. WHO child growth standards: length/height-forage, weight-for-age, weight-for-length, weightfor-height and body mass index-for-age. Geneva: WHO; 2006.

14. Barros AJD, Hirakata VN. Alternatives for logistic regression in cross-sectional studies: 
an empirical comparison of models that directly estimate the prevalence ratio. BMC Med Res Methodol. 2003;3:21. [Acesso em: 5 Dez. 2008]. Disponível em: http:// www.biomedcentral.com/1471-2288/3/21

15. Victora C, Huttly S, Fuchs S, Olinto M. The role of conceptual frameworks in epidemiological analysis: a hierarchical approach. Int J Epidemiol. 1997;26:224-7.

16. Osório MM. Fatores determinantes da anemia em crianças. J Pediatr. 2002;78:269-78.

17. Muniz PT, Castro TG, Araújo TS, Nunes NB, Silva-Nunes M, Hoffmann EEE, et al. Child health and nutrition in the Western Brazilian Amazon: population-based surveys in two counties in Acre State. Cad Saude Publica. 2007;23:1283-93.

18. Souto TS, Oliveira MN, Casoy F, Machado EHS, Juliano Y, Gouvêa LC et al. Anemia e renda per capita familiar de crianças freqüentadoras da creche do Centro Educacional Unificado Cidade Dutra, no Município de São Paulo. Rev Paul Pediatria. 2007;25:161-6.

19. Silva DG, Priore SE, Franceschini SC. Fatores de risco para anemia em lactentes atendidos nos serviços públicos de saúde: a importância das práticas alimentares e da suplementação com ferro. J Pediatr. 2007;83:149-56.

20. Stekel A. Iron nutrition in infancy and childhood. New York: Raven; 1984.

21. Garcia MT, Granado FS, Cardoso MA. Alimentação complementar e estado nutricional de crianças menores de dois anos atendidas no Programa Saúde da Família em Acrelândia, Acre, Amazônia Ocidental Brasileira. Cad Saude Publica. 2011:27;305-16.

22. Oliveira MA, Osório MM, Raposo MC. Fatores socioeconômicos e dietéticos de risco para a anemia em crianças de 6 a 59 meses de idade. J Pediatr. 2007;83:39-46.

23. Monteiro CA, Szarfarc SC, Mondini L. Tendência secular da anemia na infância na cidade de São Paulo (1984-1996). Rev Saude Publica. $2000 ; 34: 62-72$.
24. Miglioli TC, Brito AM, Lira PIC, Figueroa JN, Filho MB. Anemia no binômio mãe-filho no Estado de Pernambuco. Cad Saude Publica. 2010;26:1807-20.

25. Melo-Reis PR, Araújo LM, Dias-Penna KG, Mesquita MM, Castro FS, Costa SH. A importância do diagnóstico precoce na prevenção das anemias hereditárias. Rev Bras Hematol Hemoter. 2006;28:149-52.

26. Lacerda E, Cunha AJ. Anemia ferropriva e alimentação no segundo ano de vida no Rio de Janeiro, Brasil. Rev Panam Salud Publica. 2001;9:294-301.

27. Instituto Brasileiro de Geografia e Estatística. Pesquisa de Orçamentos Familiares - 20022003: Análise da disponibilidade domiciliar de alimentos e do estado nutricional no Brasil. IBGE: Rio de Janeiro; 2006.

28. DeMayer EM. Preventing and controlling iron deficiency anemia through primary health care: a guide for health administrators and programmer managers. Geneve: WHO; 1989.

29. Hurrell RF. Biovailability of iron. Eur J Clin Nutr. 1997;51:54-8.

30. Stulbach TE. Avaliação do Programa Nacional de Suplementação de Ferro no controle da anemia, em crianças de 6 a 24 meses, assistidas nos centros de educação infantil do município de Guarujá [Tese]. São Paulo: Faculdade de Saúde Pública da Universidade de São Paulo; 2009.

31. Bertolini GA, Vitolo MR. Baixa adesão à suplementação de ferro entre lactentes usuários de serviço público de saúde. Pediatria. 2007;29:176-82.

32. Azeredo CM, Silva LS, Franceschini SCC, Sant'ana LFR, Ribeiro RCL. Implantação e impacto do Programa Nacional de Suplementação de Ferro no município de Viçosa - MG. Cienc Saude Colet. 2011;16;4011-22.

33. Secretaria Municipal de Saúde. Relatório Anual de Gestão: Exercício de 2010. Rio Branco, 2011. [Acesso em: 03 mai 2012]. Disponível em: http://www.riobranco.ac.gov.br/v4/images/ stories/2011/J ul h o _ 2011/ relatorio_gestao_2010_semsa.pdf. 